\title{
Patient satisfaction and efficacy of accent high-intensity focused ultrasound for face lifting
}

\author{
Hamid Zandvakili ${ }^{1 *}$, Ramin Rafiei Hamid ${ }^{1}$ and Reza Chabok ${ }^{2}$ \\ Department of Biomechanics Engineering, College of Biomedical Engineering, Science and Research Branch, \\ Islamic Azad University, Tehran, Iran ${ }^{1}$ \\ NIH Resource Center for Medical Ultrasonic, Transducer Technology, Department of Biomedical Engineering, \\ University of Southern California, Los Angeles, United States ${ }^{2}$
}

Received: 6-June-2016; Revised: 13-August-2016; Accepted: 16-August-2016

(C)2016 ACCENTS

\begin{abstract}
High-intensity focused ultrasound (HIFU) method treatment has recently erosed in response to the increasing demand for skin lifting and tightening. This study was aimed at evaluating the patient's satisfaction with HIFU treatment for lifting in different areas of the face. Twenty five Iranian women patients with facial wrinkle and laxity were analyzed after a single session of HIFU treatment. Two independent, blinded clinicians and patients evaluated the clinical improvement in seven areas of the face by comparison of standardized photographs obtained before, and at two months after treatment. Assessment of satisfaction and adverse effects of treatment were done by using questionnaires. Results showed that significant differences $(p<0.05)$ between the physician and patients' satisfaction about lifting. HIFU could be an effective and non-invasive procedure that can be used to improve facial lifting in Iranian skin. If more sessions to be used in the patients more likely it will be effective.
\end{abstract}

Keywords

High-intensity focused ultrasound, Face lifting, Patient's satisfaction, Iran.

\section{Introduction}

With increased life expectancy as a result of public and personal hygiene, prevention and treatment of skin aging are necessary for some people. As human gets older, skin and it's under structural tissues constantly get ageing process. Typically, number of fibroblast on the skin decreases and collagen synthesis also decreases and functions and numbers of many skin appendages are also dropped. For under the tissues of the skin that maintain the face contours also form laxity due to the aging process. Face and scalp are composed of several layers and these can be specifically composed into five standard layers: Skin, Subcutaneous layer, Musculoaponeurotic layer (SMAS: Superficial MusculoAponeurotic System), Loose areolar tissue (spaces and retaining ligaments), fixed periosteum and deep fascia. So far in the laser treatment area of dermatology, the epidermis and dermis were the main interested therapeutic areas for the skin treatment.

\footnotetext{
*Author for correspondence
}

However, collagen remodelling on deep dermis slightly helps to regain some degree of skin elasticity but it is difficult to improve its own fundamental face contour [1].

About history of face lifting \& tightening the Latin word 'Rejuvenation'is the compound word of ' $\mathrm{Re}^{\prime}$ and 'Juvenile' which can be interpreted 'as a kid again' or 'be young again'. Rejuvenation has historically always been a concern for the human. The development of laser and other medical equipment for rejuvenation can be classified in many different ways but can be largely categorized in five: 1-Ablative resurfacing with lasers, 2-Photo rejuvenation with intense pulsed light (IPL), 3Rejuvenation with electrical current radiofrequency (RF), 4-Rejuvenation with fractional laser, 5- New generation rejuvenation with HIFU [1].

When the laser technology was not developed in old days, ablative laser or chemical peeling was used for face lifting [1]. Recently, HIFU was explored as a new treatment modality for skin tightening and rejuvenation [2,3]. High intensity focused ultrasound 
can produce small, micro-thermal lesions at precise depths in the dermis up to the fibromuscular layer, causing thermally induced contraction of collagen and tissue coagulation with subsequent collagenesis, while sparing the epidermis [4,5]. Many different laser and other light-based systems have been developed and evaluated for their capability to reverse photo damage and age-associated rhytides, a process referred to as photo rejuvenation[6,7]. Although ablative lasers remain the gold standard for photodamaged skin rejuvenation, their use is associated with side effects as well as a prolonged and unpleasant posttreatment downtime $[1,8]$. Thus, in recent years, non-invasive and non-ablative procedures have attracted attention with the hope of achieving skin tightening effects close to those of ablative lasers while avoiding long recovery times and potential complications [9-11]. Nonablative rejuvenation (NAR) devices have been designed to induce thermal injury within the dermis without epidermal damage [12-14]. However, laser energy can be diffracted, absorbed, or scattered, resulting in suboptimal energy penetration $[15,16]$.

One of the recent advances in non-invasive skin tightening is the use of HIFU. This can produce small, micro-thermal lesions at precise depths in the mid to deep reticular dermis up to the fibromuscular layer, causing thermally-induced contraction of collagen and tissue coagulation with subsequent collagenesis, while sparing the epidermis[17,18]. Recently a lot of concern about facial wrinkles has been created in the community. About this problem, various treatments, including ,microdermabrasion, fractional laser, and radiofrequency, have been proposed for the treatment of facial wrinkles and laxity; however, the ideal treatment option has yet to be identified $[4,15,19,20]$. Recently, ultrasound was introduced as a new treatment for therapeutic and cosmetic purposes $[2,4]$. HIFU may be the best example of this technique. White et al reported the first dermatologic, aesthetic use of HIFU in 2008, and HIFU was approved by the food and drug administration in 2009 for use in brow lifting [18]. Currently, it is being used for facial rejuvenation, lifting, tightening, and body contouring, which are considered 'off-label' use [10]. The principle of HIFU is to induce cellular damage and volume reduction of the target area selectively by means of coagulation by generating instant microthermal lesions through the accumulation of high-frequency ultrasound beams at the specific tissue site without any damage to the epidermis and adjacent issue [21]. But so far, few studies have confirmed the use of this method. The results in the study [19] showed that macroscopically, UVB-induced hyperpigmentation was significantly reduced two weeks after HIFU with $0.2 \mathrm{~J} / \mathrm{cm}^{2}$, and three weeks after HIFU with $0.1 \mathrm{~J} / \mathrm{cm}^{2}$. Histopathologically, the heavy deposition of melanin in the epidermis induced by UVB exposure was reduced three weeks after HIFU irradiation [19].

The aim was to compare neocollagenesis and neoelastogenesis in each layer of the dermis after each session of HIFU and monopolar RF in [3]. They analyzed the area fraction of collagen and elastic fibers using the Masson's Trichrome and Victoria blue special stain, respectively, before and after two months of treatments. Histometricanalyses were performed in each layer of the dermis, including the papillary dermis, and upper, mid, and deep reticular dermis. Results showed that Monopolar RF led to neocollagenesis in the papillary dermis, and upper, mid, and deep reticular dermis, and neoelastogenesisin the papillary dermis, and upper and mid reticular dermis. Among these treatment methods, HIFU showed the highest level of neocollagenesis and neoelastogenesis in the deep reticular dermis $[3,20]$.

Given that very few studies have been done HIFUmethod for face lifting purpose of this study was Patients' satisfactions and efficacy of accent HIFU for face lifting.

\section{Materials and methods}

This research is a cross-sectional study and was conducted in two laser center with HIFU in Tehran. Patients who have recently had a history of skin rejuvenation excluded. In our study there were 25 patients women 35-50 years (Mean $\pm \mathrm{SD}=42.56 \pm$ 4.02). All patients were consented for the procedure. The major complaint of patients was that they all tend to lift sagging facial skin in the cheeks and eyebrows. All patients were banned for 72 hours from ingestion of drugs such as NSID, aspirin, corticosteroids, heparin, vitamin $\mathrm{K}$ and vitamin $\mathrm{E}$. In this study, all of them didn't any attempt to face lifting with HIFU or similar technology in last year. Of the 25 participants in the study there were only 6 people unbearable pains. In no case was found bruises. They were treated with a HIFU face lifting device (Doublo from Hironiccompany made by South Korea) to the entire face, except for the nose and eyes, by using the following probes: Power is in the range of 0.8 to 1.2 $\mathrm{MHz}$ according to diagnosis and patient needs set in all patients. Pitch was considered $2-\mathrm{mm}$ in all patients. 
From 400 to 450 shots were delivered according to the size of the face. Standardized photographs of frontal and $45^{\circ}$ angle views, along with profiles from each side, were obtained before, and 2 months after the treatment. For comparison and record comments pre- and post-treatment photographs were printed out and organized side by side to be reviewed by physician and patients. Information collected through
SPSSGraphic18.0 (SPSS INC., Chicago, IL) software and Chi-square test analyzed in $\mathrm{p} \leq 0.05$.

\section{Results}

Patient and physician's satisfaction for face lifting are given in Figure 1.

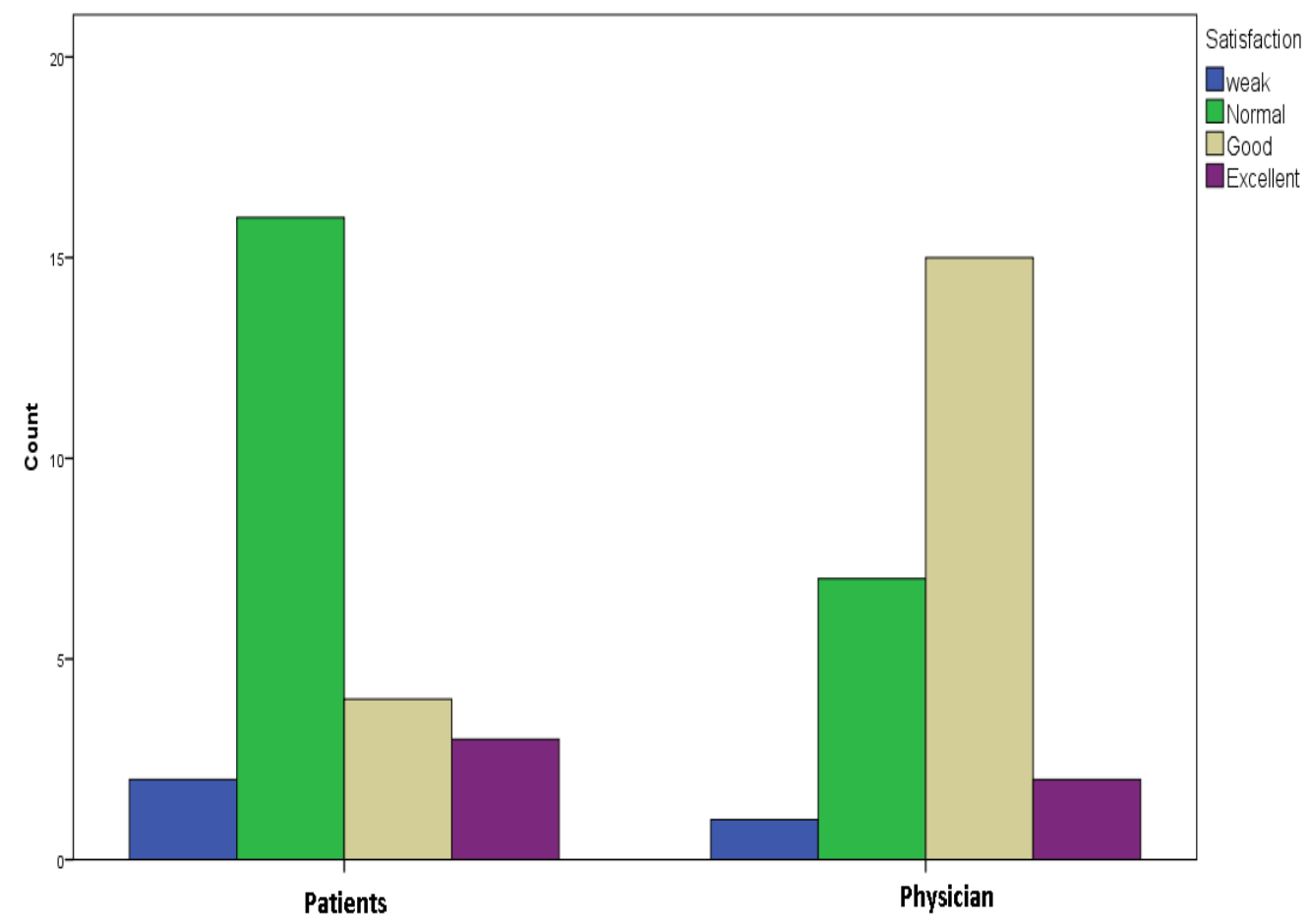

Figure 1 Patient and physician's satisfaction about face lifting

16 patients were assessed their face lifting as moderate and normal, 4 people good, 3 people excellent and only 2 people assessed that weak. Physician were evaluated 15 patients good in face lifting, 7 people normal, 2 people excellent and only for one person assessed weak. Chi-square test method is shown in the Table 1 and compares patient and physician satisfaction about face lifting with HIFU.

Table 1 Chi-square test

\begin{tabular}{cccc} 
& Value & Df & Sig \\
\hline Pearson & 10.423 & 3 & $0.015^{*}$ \\
Chi-square & & & \\
\hline
\end{tabular}

*p $\leq 0.05$

The results showed that patient and physicians' perspectives about face lifting with HIFU are statistically meaningful differences $(\mathrm{p} \leq 0.05)$.

\section{Discussion}

Both intrinsic and extrinsic aging processes contribute to aesthetic changes. Advances in the understanding of skin biology have led to the development of a number of technologies for rejuvenating the skin. Current treatment modalities include ablative and non-ablative lasers, radiofrequency devices, and ultrasound devices [2, $22,23]$.

Recently, HIFU treatment has received proper medical attention as a 'high-tech' way to improve face lifting $[3,4,10]$. To date, few articles have demonstrated the efficacy of HIFU face lifting and devices. HIFU was a safe and effective modality for facial skin tightening [4]. Another recent study showed that HIFU could also be used safely and effectively to improve the skin texture and contour of 
the upper arms, extensor knees, and medial thighs [24]. In [5], it is reported the clinical and histopathologic changes after HIFU treatment, and suggested that HIFU was a safe, effective, and noninvasive procedure that can be used to tighten the facial skin of Asian people[5]. In this study we have divided the face into several areas and then evaluated face after HIFU treatment with clinicians and patients.

In this study, significant difference was observed between and patient's satisfaction with face lifting ( $p$ $<0.05$ ). Physician and patients' satisfaction with the clinical effects of HIFU seemed not similar. Physician satisfaction levels more than patients. This suggests that patients need more time than two month to better see changes in their faces. Hence more studies are needed to be done at greater intervals to attract patient satisfaction as well as other researches. Because with increases in time of review in park et al it was observed [25]. In their study subjective satisfaction was sustained for at least 6 months. This may be because HIFU not only resulted in facial lifting and improvement of wrinkles but also in improvements in skin tone, facial contour, and subjective symptoms such as tightness or tension on the skin. This study has two limitations. First, the number of participants was limited. Second, due to the HIFU being new technique there was the possibility of adverse effect.

\section{Conclusion and future work}

We have performed facial lifting with Doublo with 35 patients older than 35 years, and observed clinical improvement and somewhat patient satisfaction after the treatment. HIFU can be safe, effective, and noninvasive option for facial lifting. Further studies should be considered with more patients conducted at multiple institutions. Additionally, there is a need for further studies using from different patients and different methods such as different shots and in combination with other methods and tools such as botulinum toxin.

\section{Acknowledgment}

We appreciated with cooperation of Dr.Sahar Atighi of Kara Darman Clinic for collecting information and monitoring in this study.

\section{Conflicts of interest}

The authors have no conflicts of interest to declare.
References

[1] Kim JH. Doublo: Clinical study of non-invasive facelifting treatment in application of HIFU. http://www.doubloterapi.com/img/uzman_gorusleri/cli nical_study_of_noninvasive_face_lifting_treatment_in_application_of_hif u_12.05.2014_466a5d6.pdf. Accessed 16 May 2016.

[2] Lee HJ, Lee KR, Park JY, Yoon MS, Lee SE. The efficacy and safety of intense focused ultrasound in the treatment of enlarged facial pores in Asian skin. Journal of Dermatological Treatment. 2015; 26(1):73-7.

[3] Suh DH, Choi JH, Lee SJ, Jeong KH, Song KY, Shin MK. Comparative histometric analysis of the effects of high-intensity focused ultrasound and radiofrequency on skin. Journal of Cosmetic and Laser Therapy. 2015; 17(5):230-6.

[4] Alam M, White LE, Martin N, Witherspoon J, Yoo S, West DP. Ultrasound tightening of facial and neck skin: a rater-blinded prospective cohort study. Journal of the American Academy of Dermatology. 2010; 62(2):262-9.

[5] Suh DH, Shin MK, Lee SJ, Rho JH, Lee MH, Kim NI, et al. Intense focused ultrasound tightening in Asian skin: clinical and pathologic results. Dermatologic Surgery. 2011; 37(11):1595-602.

[6] Lee YB, Eun YS, Lee JH, Cheon MS, Cho BK, Park HJ. Effects of multi-polar radiofrequency and pulsed electromagnetic field treatment in Koreans: case series and survey study. Journal of Dermatological Treatment. 2014; 25(4):310-3.

[7] Zelickson BD, Kist D, Bernstein E, Brown DB, Ksenzenko S, Burns J, et al. Histological and ultrastructural evaluation of the effects of a radiofrequency-based nonablative dermal remodeling device: a pilot study. Archives of Dermatology. 2004; 140(2):204-9.

[8] Alexiades-Armenakas MR, Dover JS, Arndt KA. The spectrum of laser skin resurfacing: nonablative, fractional, and ablative laser resurfacing. Journal of the American Academy of Dermatology. 2008; 58(5):719-37.

[9] Dierickx CC. The role of deep heating for noninvasive skin rejuvenation. Lasers in Surgery and Medicine. 2006; 38(9):799-807.

[10] Chan HH, Yu CS, Shek S, Yeung CK, Kono T, Wei WI. A prospective, split face, single-blinded study looking at the use of an infrared device with contact cooling in the treatment of skin laxity in Asians. Lasers in Surgery and Medicine. 2008; 40(2):146-52.

[11] Gold MH. Aesthetic practice trends: tissue tightening: a hot topic utilizing deep dermal heating. Journal of Drugs Dermatology. 2007; 6(12):1238-42.

[12] Sadick NS. Update on non-ablative light therapy for rejuvenation: a review. Lasers in Surgery and Medicine. 2003; 32(2):120-8.

[13] Hardaway CA, Ross EV. Nonablative laser skin remodeling. Dermatologic Clinics. 2002; 20(1):97111. 
[14] Hohenleutner S, Hohenleutner U, Landthaler $M$. Nonablative wrinkle reduction: treatment results with a 585-nm laser. Archives of Dermatology. 2002; 138(10):1380-1.

[15] El-Domyati M, El-Ammawi TS, Medhat W, Moawad O, Brennan D, Mahoney MG, et al. Radiofrequency facial rejuvenation: evidence-based effect. Journal of the American Academy of Dermatology. 2011; 64(3):524-35.

[16] Ruiz-Esparza J. Nonablative radiofrequency for facial and neck rejuvenation. A faster, safer, and less painful procedure based on concentrating the heat in key areas: the ThermaLift concept. Journal of Cosmetic Dermatology. 2006; 5(1):68-75.

[17] Gliklich RE, White WM, Slayton MH, Barthe PG, Makin IR. Clinical pilot study of intense ultrasound therapy to deep dermal facial skin and subcutaneous tissues. Archives of Facial Plastic Surgery. 2007; 9(2):88-95.

[18] White WM, Makin IR, Barthe PG, Slayton MH, Gliklich RE. Selective creation of thermal injury zones in the superficial musculoaponeurotic system using intense ultrasound therapy: a new target for noninvasive facial rejuvenation. Archives of Facial Plastic Surgery. 2007; 9(1):22-9.

[19] Choi SY, Yoo KH, Oh CT, Kwon TR, Choi EJ, Seok $\mathrm{J}$, et at. High intensity focused ultrasound as a potential new modality for the treatment of pigmentary skin disorder. Skin Research and Technology. 2015;22(2):131-6.

[20] Chan NP, Shek SY, Yu CS, Ho SG, Yeung CK, Chan HH. Safety study of transcutaneous focused ultrasound for non-invasive skin tightening in Asians. Lasers in Surgery and Medicine. 2011; 43(5):366-75.

[21] Brobst RW, Ferguson M, Perkins SW. Ulthera: initial and six month results. Facial Plastic Surgery Clinics of North America. 2012; 20(2):163-76.
[22] Shapiro SD, Eros Y, Abrahami Y, Leviav A. Evaluation of safety and efficacy of the TriPollar technology for treatment of wrinkles. Lasers in Surgery and Medicine. 2012; 44(6):453-8.

[23] Bloom BS, Emer J, Goldberg DJ. Assessment of safety and efficacy of a bipolar fractionated radiofrequency device in the treatment of photodamaged skin. Journal of Cosmetic and Laser Therapy. 2012; 14(5):208-11.

[24] Alster TS, Tanzi EL. Noninvasive lifting of arm, thigh, and knee skin with transcutaneous intense focused ultrasound. Dermatologic Surgery. 2012; 38(5):754-9.

[25] Park H, Kim E, Kim J, Ro Y, Ko J. High-intensity focused ultrasound for the treatment of wrinkles and skin laxity in seven different facial areas. Annals of Dermatology. 2015; 27(6):688-93.

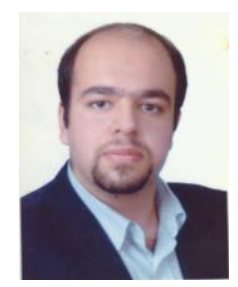

Hamid Zandvakili received his B.S. degree in mechanical engineering from Shahid Bahonar University of Kerman in 2005, and received M.S. degrees: in biomedical engineering from Department of Bioelectric Engineering, College of Biomedical Engineering, Tehran Science and Research Branch, Islamic Azad University, Tehran, Iran in 2014, and He finally Studies his Ph.D. degree in biomedical engineering at Department of Bioelectric Engineering, College of Biomedical Engineering, Tehran Science and Research Branch, Islamic Azad University, Tehran, Iran now His interests include high frequency ultrasonic transducers and arrays, and digital methods for fabrication of piezoelectric materials and composites for medical imaging applications.

Email: hamid.zandvakili@gmail.com 